\title{
Bifurcation transition in a system of two microwave oscillators during coherence destruction
}

\author{
Sergey Novikov* \\ Tomsk State University, Tomsk 634050, Russian Federation
}

\begin{abstract}
The fine structure of bifurcation changes of oscillatory regimes in a system of two microwave oscillators in the region of mutual resonant strong coupling is experimentally investigated. Briefly discusses the methods for circuit implementation of strong resonant interactions, as well as their analytical threshold, above which synchronous modes lose stability and the system goes into dynamic chaos mode.
\end{abstract}

\section{Introduction}

It is generally accepted that in the simplest system of two coupled oscillators, when their natural frequencies are close, synchronism occurs. At the same time, as our studies show, mutual synchronization is one of the options for the behavior of coupled self-oscillating systems. So, at strong resonant interactions, the coherent mode loses stability, and there is a tendency for the system to transition into disordered, chaotic motion.

Chaotic dynamics in self-oscillating structures often occurs due to the instability of some basic regular movements and is the result of specific interactions of dynamic variables of the system, which are the components of the movement. For coupled oscillators these basic movements are synchronous modes. Therefore, the search and physical interpretation of direct relationships between the stability indicators index of the basic movements and the system parameters become important in the study of dynamic processes. The physical notion on the stability (or instability) of certain movements in dynamical systems can be useful for understanding the bifurcation transformations of their oscillatory regimes.

The most effective method for studying the stability of dynamical systems, often giving analytically solutions, is to study local stability. In $[1,2]$ theoretically and experimentally it is shown that the synchronous mode in a system of two identical oscillators with "strong" resonance properties of mutual coupling loses its stability. Later in the paper [3], we have formulated a fairly simple and universal criterion of the coherence destruction and of transition to chaotic dynamics. The control parameters of this criterion are the parameters of the passive coupling circuit, available for wide change. A significant feature of the model is that each of self-oscillators in autonomous mode is absolutely stable and singlefrequency system, and the generators can be identical.

*Corresponding author: nss@sibmail.com 
As experience shows [5-8], the oscillatory modes of a coupled oscillators system at changing control parameters during the destruction of coherence and transition to chaotic dynamics demonstrate bifurcation transformations, which have both characteristic features of classical scenarios and features associated with basic synchronous oscillations. The study of these transitions on the basis of ideas about their stability or instability makes it possible to compose a phenomenological picture of the behavior of coupled self-oscillators system, and to identify characteristic dynamic signs of oscillatory processes in chaotic modes.

In the present work the fine structure of bifurcation changes in oscillatory regimes of a two microwave oscillators system in the region of strong resonance interconnection during coherence destruction is experimentally investigated.

\section{Coherence destruction criterion}

The system of two self-oscillators at general form shown in Fig.1. Self-oscillators with a resonant active elements $S_{k}\left(U_{k}\right)$ and resonance system $y_{k}(j \omega)$ are coupled through passive four-pole $\mathrm{Y}$, which contains a common load or other dissipative elements. At the close of

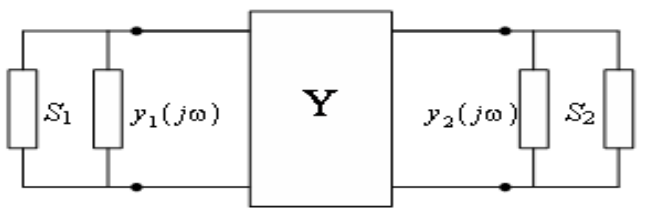

Fig. 1. System of two coupled self-oscillators. the partial frequency synchronous oscillations can exist in the system; and in case of equality of the frequencies and the complete symmetry of system they correspond to the phase difference $\Delta \varphi=0, \pi$; these oscillations are basic.

In the paper [4] it was shown that the coupling conductivity of self-oscillators with minimal restrictions in general is described by the formula:

$$
y_{12}=\frac{-2 s}{\left(\cos \alpha_{S}-2 s \cos \theta\right)+j \sin \alpha_{S}} g_{0},
$$

Where $s$ and $\alpha_{S}$ are module and argument of the scattering matrix element $S_{12}=s \exp \left(-j \alpha_{S}\right)$; $g_{0}$ - corresponds to the wave conductivity of the input lines of the four-pole Y; $\theta-$ electrical asymmetry parameter, its meaning is defined below. The value lies in the range (0-0.5). The upper value corresponds to the absence of any other dissipative elements in the Y- circuit, with the exception of the common matched load.

If we assume that the phase parameter $\alpha_{S}$ depends on the frequency, it follows from (1) that the mutual coupling parameter $y_{12}(j \omega)$ has significant resonant properties. These properties are described by parameter

$$
C_{12}=d\left(\operatorname{Im} y_{12}(j \omega)\right) /\left.d \omega\right|_{\omega_{0}} .
$$

It is essential that in regions near $\alpha_{S} \approx 2 \pi, 4 \pi, \ldots$ at $s \rightarrow 0.5$ and $\theta \rightarrow 0.5$ the parameter $C_{12}$ is positive $\left(C_{12}>0\right)$ and theoretically reach arbitrarily large values. The analytical criterion for the destruction of coherence for symmetric systems formulated in [3] has the form:

$$
C_{12}>C,
$$

where $C_{1}=C_{2}=C$ - capacity of oscillatory systems $y_{k}(j \omega)$ of self-oscillators. When performing (3) synchronous in-phase oscillations (basic mode), as well as oscillations in the adjacent region of nonzero phase differences lose stability. Instability in this case, as shown in [3], develops simultaneously in two directions. These directions of phase space correspond to opposite phase perturbation of oscillation of self-oscillators and opposite disturbances of their amplitudes. 


\section{Coupling circuit with resonant properties and control parameters}

Figure 2 shows the profile of the frequency characteristics of the coupling parameter $y_{12}(j \omega)$ corresponding to formula (1). A strong resonant coupling of type (3), which causes instability of in-phase and close oscillations, is realized in narrow frequency intervals lying inside the region $\operatorname{Re} y_{12}<0$, where the derivative of the imaginary part is positive: $C_{12}>0$. The inset in Fig. 2 shows the simplest version of the coupling circuit, fully demonstrating the indicated resonance properties. In accordance with this scheme, self-oscillators are connected to each other and to a common load $g_{\text {н }}$ by transmission line segments with electric lengths $\theta_{1}$ and $\theta_{2}$ and wave conductivity $g_{0}$. For this circuit $\alpha=\theta_{1}+\theta_{2} \equiv \theta_{\Sigma} \sim \emptyset \tau$, where $\tau$ is the signal propagation time in the channel; $\theta=\theta_{2}-\theta_{1}$ is the asymmetry parameter; $g_{\mathrm{H}}=2 g_{0}$ is

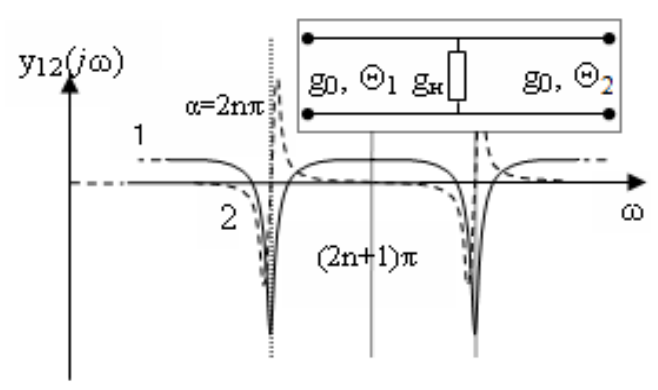

Fig. 2. Resonance properties of the coupling conductivity; $1-\operatorname{Re} y_{12}, 2-\operatorname{Im} y_{12}$. the conductivity of the matched total load.

The listed parameters are easily changed in a real experiment and allow controlling the value of parameter $C_{12}$ over a wide range. Thus, introducing linear losses into transmission line segments directly reduces the value of the parameter $s$, and, as follows from (1), expands the resonance regions and reduces the value of $C_{12}$. A similar control is realized by introducing the asymmetry $\theta$. An effective tuning is the tuning of the natural frequencies of the oscillators: precision frequency control in the field of resonance coupling allows one to reveal fine features of bifurcation changes in the oscillatory modes of the system directly during the transition to chaotic dynamics.

The indicated strong resonant properties of oscillators mutual coupling are also realized in circuits that are combinations of transmission line segments and loads: antisymmetric circuits, circuits with many loads in the wave channel, and also in circuits including reactive elements [7].

\section{Experimental study of bifurcation transitions}

Fig. 3 shows the studied symmetric circuit with the measuring equipment. Microwave selfoscillators have identical designs as a single circuit boards with transistors $T_{1}, T_{2}$ of type KT640A2. Variable-capacitance diode $D_{1}, D_{2}$ of type АП320 included in the strip resonators of oscillators and provide frequency tuning in the range $2.9-3.5 \mathrm{GHz}$. It is important to emphasize, that self-oscillators at autonomous regime in the entire tuning range exhibit a regular single-frequency mode. Self-oscillators connected to each other by a T-joint; it is realized on air asymmetric strip line sections. This provides a small linear losses of the wave coupling channel and proximity of the parameter $s$ to the limiting value of 0.5 , which guarantees the fulfillment of the conditions (3). The parameter s was varied between $0.5-0.38$ by introducing thin absorbent paper into the air gap of the line (Fig.4). Generator boards and coupling circuit connected via standard coaxial-strip joints. 


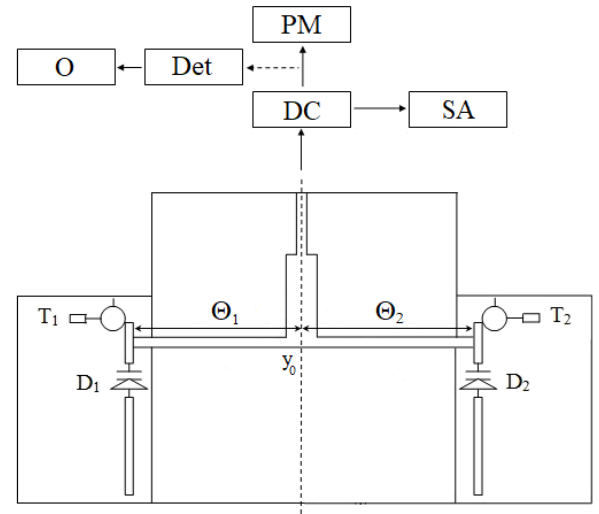

Fig. 3. The experimental scheme.

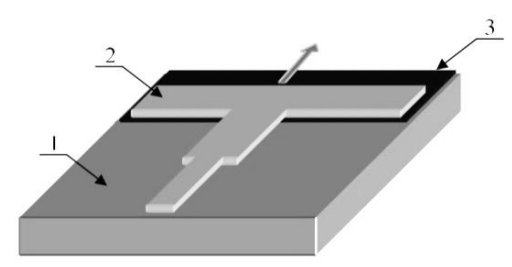

Fig. 4. Scheme introducing of losses into coupling channel; 1 - metal base, 2 - strip line sections, 3 - thin absorbent paper.

summed up in the total load.

Fig. 5 reflects the results of the experiment with a sequential (top to bottom) decreasing losses in the coupling channel, that is, with increasing parameter $s$; the natural frequencies of oscillators in this case correspond to the center of the resonance region (approximately $2920 \mathrm{MHz}$ ) and differ by $20-30 \mathrm{MHz}$. At maximum losses $(\mathrm{s} \approx 0.38)$, the almost in-phase synchronous mode is stable. The power level in the total load in this case (taking into account the introduced losses) is equal to the sum of the power of the generators. As losses decrease, in-phase regime loses stability. First, a weak instability of the periodic modulation type arises. Further, the degree of instability increases, and depth of [auto]modulation of the generators amplitudes and the phase difference increases; the modulation period takes about $20 \div 25$ periods of high-frequency oscillations. The spectrum of the output signal remains discrete, but becomes more saturated, which reflects the nonlinear nature of oscillations interaction.

With some fairly small losses, the modulation regime acquires the features of intermittent chaos. The "laminar" stage is characterized by a long, almost unchanged level of self-modulation of the output signal. The corresponding waveform in Fig. 5 taken during the sweep is $500 \mathrm{~ns} /$ div versus $50 \mathrm{~ns} /$ div for others; it was possible to fix in experiments the duration of the laminar stages up to several hundred periods of modulation. A wide signal swing indicates a significant change in the phase difference of the oscillations. Special studies (see [8]) show that changes of the oscillators amplitude envelopes in the laminar stage occur in antiphase; this fact is in agreement with the theoretical prediction of the instability of the amplitude directions. 


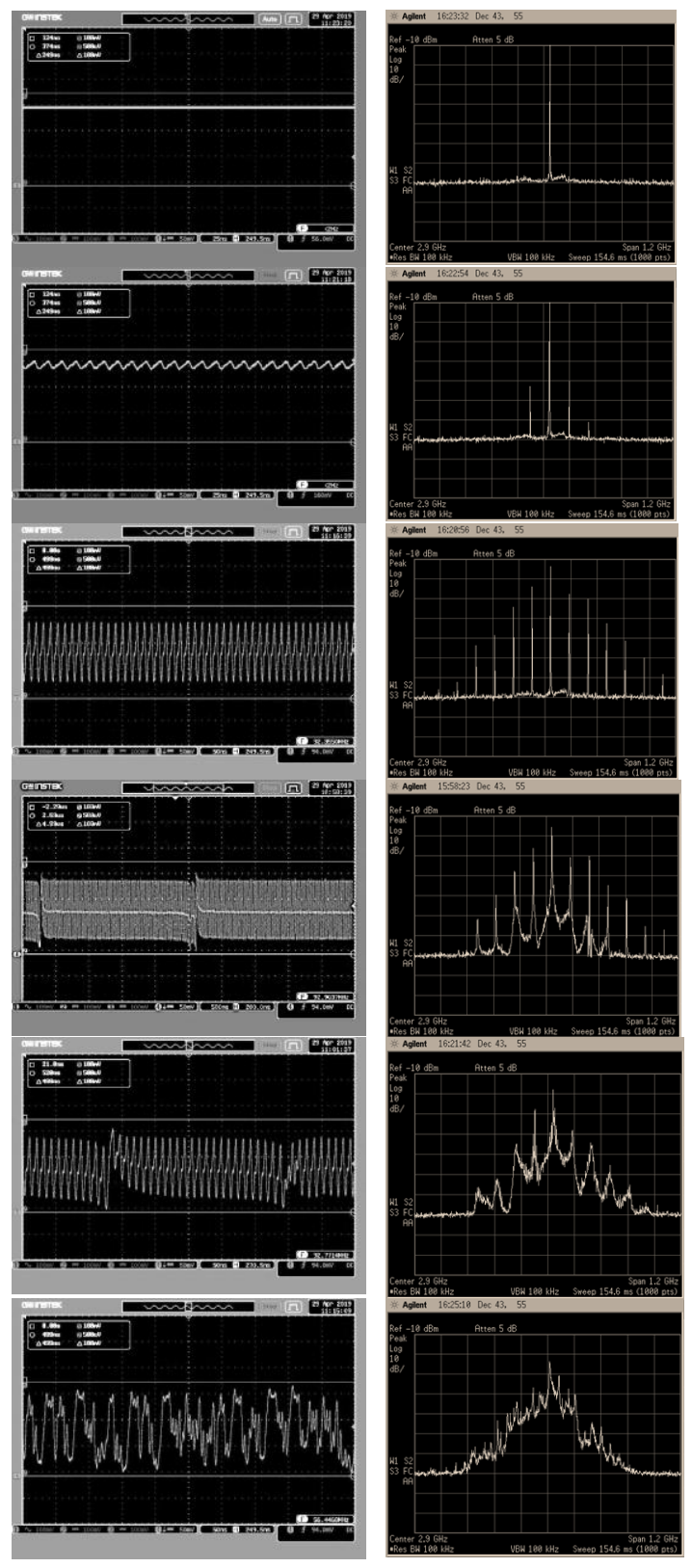

Fig. 5. Envelopes and spectra of output signal while reducing losses of coupling channel at oscillators frequencies detuning 20-30 MHz.
At the end of the laminar stage, when the level of the output signal is almost zero (that is the highfrequency oscillations are almost in antiphase), a rapid "turbulent" return of oscillations to the in-phase region occurs. The turbulent stage introduces an element of irregularity into the dynamics: the oscillation spectrum, together with discrete components, acquires a characteristic noise pedestal. Further, with an increase in the parameter $s$, the duration of the laminar stage decreases.

Finally, the laminar stage disappears and the processes of amplitude and phase difference changing lose their periodicity signs. It may notice that the envelope of the output signal contains repeating fragments, which indicates the dynamic nature of the processes. Apparently, a set of "discrete peaks" of the spectrum corresponds to these fragments.

Here it should be noted a rather obvious, but at the same time, important from a phenomenological point of view, feature of the model under consideration. When interpreting the experimental results we use as dynamic variables not the instantaneous values of the $\mathrm{HF}$ oscillations, but their envelopes, that is, their amplitudes and phase differences. This view is consistent with the formalism of the mathematical apparatus of slowly varying complex amplitudes and the ensuing consequences and physical notion about the stability of amplitudes and phases.

In a similar experiment with a small difference in the frequencies of oscillators (about 2-3 $\mathrm{MHz}$ ) the transition to chaos occurs more rigidly (Fig. 6). With decreasing losses the process of intermittency is practically absent. In the initial state (upper pictures), the system with a high degree of periodicity generates auto-transitions between in-phase and complex modulated oscillations. Therefore, the spectrum has a high level of discrete components. 

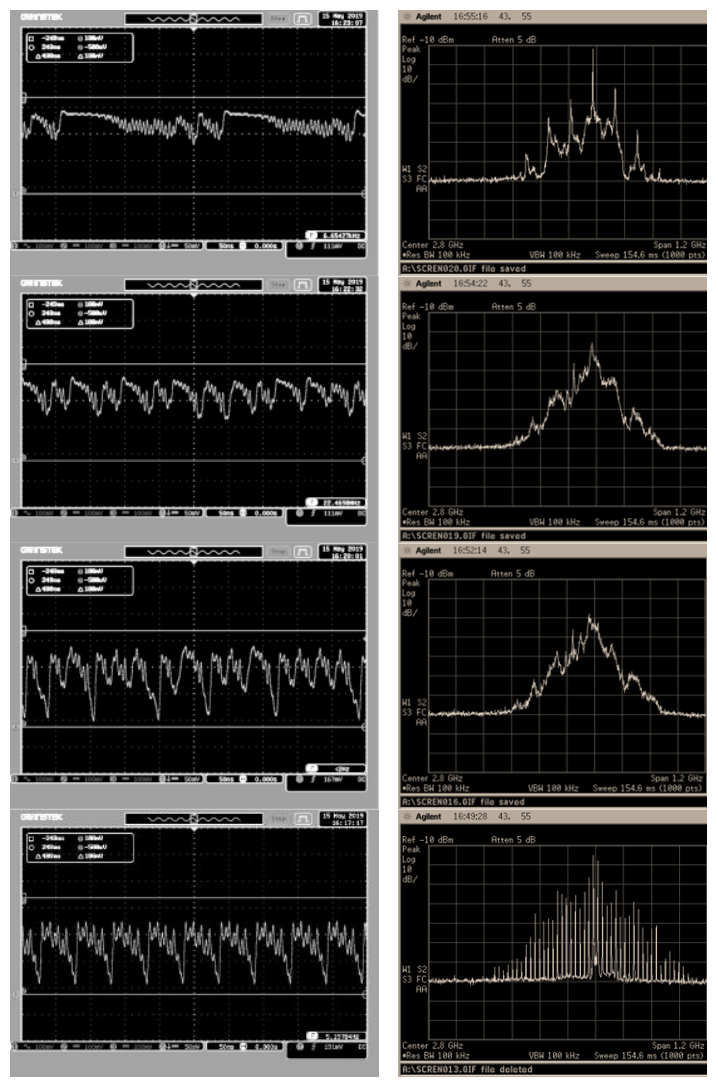

Fig. 6. Envelopes and spectra of output signal while reducing losses of coupling channel at small oscillators frequencies detuning 2-3 MHz.
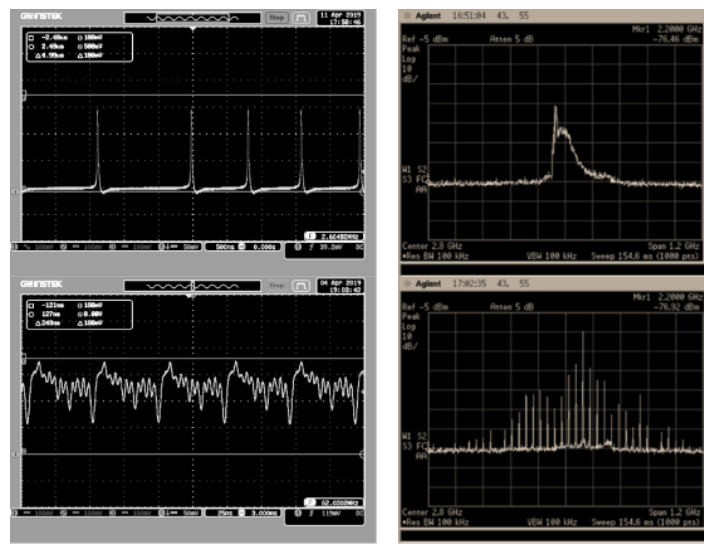

Fig. 7. Auto-transitions on the boundary of stability.

Further, with decreasing losses inphase intervals decrease, the intensity of changes in the envelope of output signal and the degree of its nonperiodicity increase. The level of discrete spectrum peaks decreases. The described sequence of oscillation transformation can be interrupted by variants of periodic modes with a complex law of amplitude-phase modulation (lower pictures). The spectrum of such oscillations is very saturated and discrete. The analysis of time realizations in Fig. 6 illustrates the deterministic properties of the system under study.

The characteristics of Fig. 7 were taken during the detuning of the oscillator frequencies from the coupling resonance $(2850 \mathrm{MHz})$; the regime of broadband chaos is not realized. With such a detuning, the coupling conductivity contains a significant reactive component, which, as is known, can cause the stability of antiphase (or in-phase) oscillations. With a mutual detuning of the generators frequencies of the order of $20 \mathrm{MHz}$, it was possible to fix the pulsed auto-transitions. For very long (about $1 \mu \mathrm{s}$ ) time intervals the system operates in antiphase mode. Since the system is located at the stability boundary, random fluctuations irregularly transfer it to the region of in-phase oscillations, which, are unstable. The system as it were produces short non-periodic radio pulses having a continuous spectrum.

These auto-transitions are localized in a very narrow frequency range (1-2 $\mathrm{MHz})$, so for their registration very precise control of generators frequencies is necessary. When the oscillator frequencies are shifted, alternating synchronous antiphase oscillations and oscillations with a complex periodic self-modulation law are observed; the latter can have a very saturated discrete spectrum (Fig. 7).

In the next experiment, a small asymmetry of the order of $\theta \approx(8-10)^{\circ}$ was introduced into the system. The introduction of asymmetry, as mentioned above, extends the frequency 

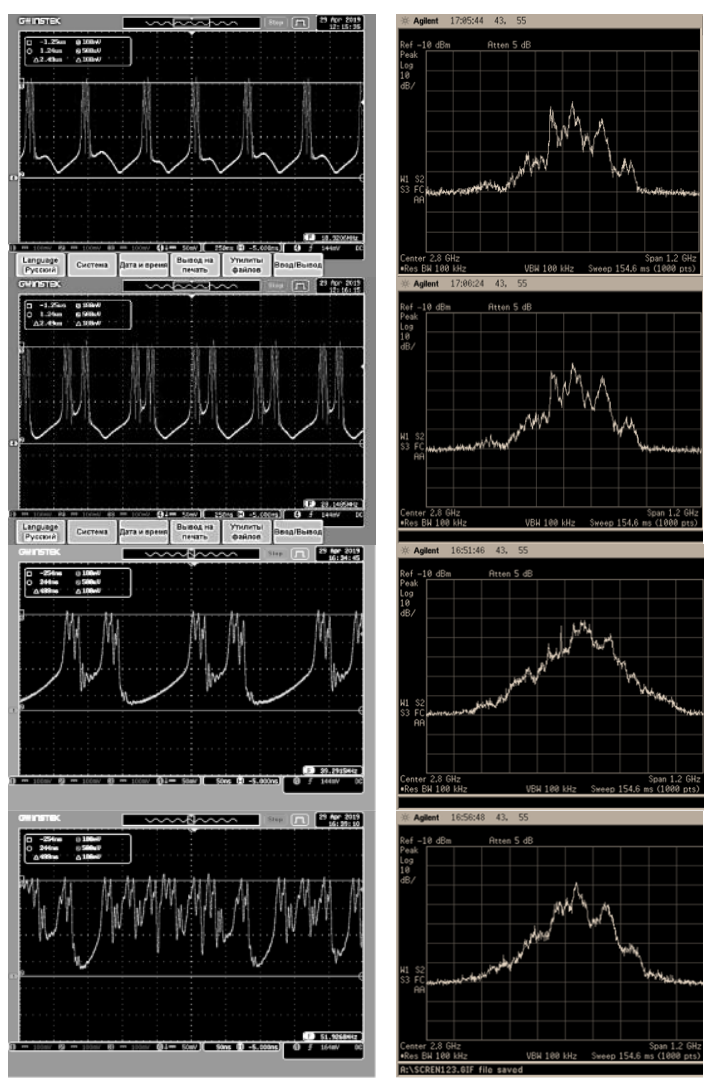

Fig. 8. Envelopes and spectra of output signal in the auto-transition mode with the introduction of asymmetry.
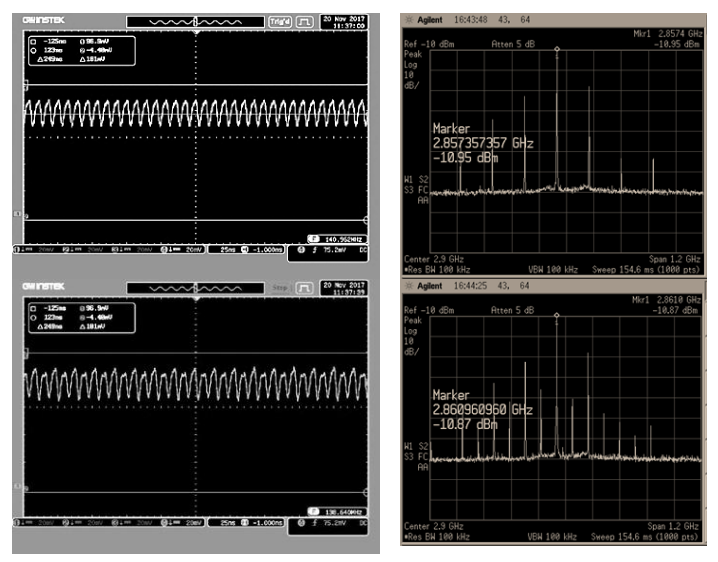

Fig. 9. The cascade of bifurcations of doubling the envelope modulation period. range where the criterion (3) for the destruction of coherence can be fulfilled. Figure 8 shows the oscillograms and spectra taken with a sequential decrease in the mutual detuning of the natural frequencies of the self-oscillators (from 25-30 $\mathrm{MHz}$ or less) also during the detuning from the coupling resonance $(2850 \mathrm{MHz})$.

As in the previous version, the generators operate for almost antiphase for long time intervals (about $0.3 \mu \mathrm{s}$ ). Then, the oscillations are automatically transferred to the in-phase region, where dynamic instability develops. The temporal extent of this regime is $25-30 \mathrm{~ns}$, after which the system returns to the region of antiphase oscillations. Thus, the system being on the stability boundary, as it were generates radio pulses with chaotic filling. As the oscillators frequencies approach, the pulses fall apart, and the intervals between them decrease. It is very characteristic that the repeating "chaotic impulses" have an almost identical structure, but are nonperiodic.

Finally, with small detunings (5$10 \mathrm{MHz}$ ), a completely developed chaotic process with a continuous spectrum is formed. The described auto-transitions are observed in a narrow - not more than $1 \mathrm{MHz}$ frequency range of one of the generators and are registered at very accurate frequency fixation using highly stable precision adjustable sources for variable-capacitance diodes.

The system under consideration is also characterized by a transition to dynamic chaos through bifurcations of doubling the envelope modulation period. These bifurcations are observed when the frequencies of the oscillators approach each other in the band of mutual detunings of the order of 10-15 MHz. Figure 9 shows a typical sequence of changes in the envelope of the signal of the total load and its spectrum 

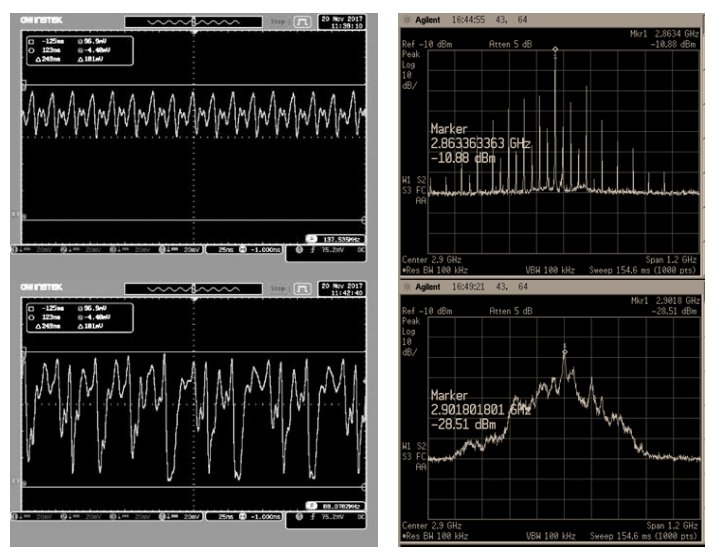

Fig. 10. The cascade of bifurcations of doubling the envelope modulation period (continued). in the indicated scenario. Each bifurcation is accompanied by the appearance of intermediate components in the initial spectrum. Usually, after two, less often - three bifurcations, a sharp transition to a chaotic regime occurs (lower pictures). The nature of the change in the envelope substantially depends on the accuracy of tuning the natural frequencies of self-oscillators to the coupling resonance. It can also be seen from the last oscillogram in Fig. 9 that the instantaneous phase difference of oscillations of selfoscillators occasionally "visits" regions with phase differences $\Delta \varphi=0, \pi$, but, due to instability, does not linger there. Here, also, within the framework of one temporal realization, a series of repeating transitions from almost in-phase to almost antiphase oscillations is visible. Individual doubling bifurcations without transition to chaotic dynamics are observed with a significant detuning of the oscillator frequencies from the coupling resonance: up to $100-150 \mathrm{MHz}$.

\section{Conclusion}

Thus, the system of two microwave oscillators in the region of strong resonance coupling shows a variety of bifurcation changes in the oscillations and their spectra during the destruction of coherence and the transition to dynamic chaos. It is shown that in the research model classical bifurcation scenarios of transition to chaos are realized, their phenomenological features are considered. It was experimentally shown that in the structure of chaotic oscillations there are repeating fragments of oscillatory motion, which indicates their dynamic nature. The processes of automatic transitions between elements of synchronous movements and chaotic dynamics were discovered and described; autotransitions are realized in a very narrow range of parameters (frequencies) and, apparently, arise at the stability boundary of basic synchronous modes.

\section{References}

1. S. S. Novikov, Dynamic and static instabilities of coherent self-oscillating system with controlled couplings, Proc. 14-th Symposium on High Current Electronics, Tomsk, Russia, pp. 427-430 (2006)

2. S. S. Novikov, A.A. Usjukevitch, Chaotic oscillation in the auto-oscillator system with resonant couplings, Proc. 16-th Int. Symposium on High Current Electronics, Tomsk, Russia, pp. 512-515 (2010)

3. S. S. Novikov, A.A. Usjukevitch, Instability of synchronous mode in a system of two coupled microwave self-oscillators, Russian Physics Journal, 55, №11, pp. 1296-1302 (2012)

4. S.A. Maidanovskij, S.S. Novikov, Symmetric and asymmetric systems of strongly coupled selfexcited oscillators, Radiotekhnika i Elektronika, 48, № 5, pp. 595-600 (2003)

5. S.S. Novikov, A.A. Usjukevitch, Destruction of the coherent mode in system of two oscillators at the strong resonant mutual couplings, Izvestiya vuz. Applied nonlinear dynamics, 20, №5, pp. 16-30 (2012) 
6. S.S. Novikov, A.A. Usjukevitch, Bifurcation transitions to the dynamic chaos mode in mutual coupled microwave oscillators system, Proc. $24^{\text {th }}$ Int. Crimean Conf. "Microwave \& Telecommunication Technology", Sevastopol, Crimea, Russia, pp.107-108 (2014)

7. S.S. Novikov, A.A Usjukevitch, Coupling circuits for microwave self-oscillator systems with chaotic dynamics, Proc. 25-th Int. Crimean Conf. "Microwave \& Telecommunication Technology", Sevastopol, Crimea, Russia, pp.525-526 (2015)

8. S.S. Novikov, Hyper-chaos Mode in the Mutual Coupled and Partual Stable Microwave Oscillators System, Progress In Electromagnetics Research Symposium - Spring (PIERS), St Petersburg, Russia, 22-25 May, pp. 1065-1069 (2017) 